\title{
Mydriasis Associated with Local Dysfunction of Parasympathetic Nerves in Two Dogs
}

\author{
Teppei KANDA ${ }^{1)}$, Kazuhiro TSUJI ${ }^{1}$, Keiko HIYAMA ${ }^{2)}$, Takeshi TSUKA ${ }^{1)}$, Saburo MINAMI ${ }^{1)}$, Yoshiaki HIKASA ${ }^{1)}$, \\ Toshinori FURUKAWA ${ }^{3)}$ and Yoshiharu OKAMOTO ${ }^{1) *}$ \\ ${ }^{1)}$ Department of Veterinary Medicine, Faculty of Agriculture, Tottori University, 4-101, Koyama-minami, Tottori 680-8553, \\ ${ }^{2)}$ Takamori Animal Hospital, 3706-2, Agarimichi, Sakaiminato, Tottori 684-0033 and ${ }^{3)}$ Department of Comparative Animal Science, \\ College of Life Science, Kurashiki University of Science and the Arts, 2640, Tsurajima-nishinoura, Kurashiki, Okayama 712-8505, Japan.
}

(Received 13 August 2009/Accepted 16 November 2009/Published online in J-STAGE 9 December 2009)

ABSTRACT. In clinical practice, photophobia resulting from persistent mydriasis may be associated with dysfunction of ocular parasympathetic nerves or primary iris lesions. We encountered a 5-year-old Miniature Dachshund and a 7-year-old Shih Tzu with mydriasis, abnormal pupillary light reflexes, and photophobia. Except for sustained mydriasis and photophobia, no abnormalities were detected on general physical examination or ocular examination of either dog. We performed pharmacological examinations using $0.1 \%$ and $2 \%$ pilocarpine to evaluate and diagnose parasympathetic denervation of the affected pupillary sphincter muscles. On the basis of the results, we diagnosed a pupillary abnormality due to parasympathetic dysfunction and not to overt primary iris lesions. The test revealed that neuroanatomic localization of the lesion was postciliary ganglionic in the first dog.

KEY WORDS: autonomic nervous system, canine, mydriasis, ophthalmology, tonic pupil.

Mydriasis and miosis are normal physiological reactions that allow the eye to adjust to the level of incoming light. Mydriasis is induced by excitation of the sympathetic nervous system by nociceptive or mental stimulation. Conversely, miosis is controlled by the parasympathetic nervous system. Therefore, dysfunction of the sympathetic or parasympathetic nerves that regulate pupil size can induce abnormal miosis or mydriasis. Sustained mydriasis can result in photophobia.

Abnormal pupil size can be caused by dysfunction of the ocular autonomic nerves. In humans, there have been many reports of Horner's syndrome, a sympathetic ocular nervous system disorder, and Adie's syndrome, a parasympathetic ocular disorder that results in miosis. [8] Horner's syndrome is a common neurological ophthalmologic disorder associated with miosis in dogs [3]. There have also been reports of pupillotonia with miosis, and mydriasis from ophthalmoparesis and dysautonomia in dogs $[2,5,6,9]$. In Japan, there have been no reports of dogs with abnormal pupil size because of autonomic nervous dysfunction.

Primary iris lesions (e.g., iris atrophy and synechia) are known causes of mydriasis. Therefore, for adequate diagnosis, veterinarians should discern whether the cause of the abnormal mydriasis is parasympathetic dysfunction or a primary iris lesion. Additionally, if the cause of mydriasis is parasympathetic dysfunction, it may be important to determine whether the parasympathetic dysfunction is local or systemic. We diagnosed 2 dogs with abnormal mydriasis induced by local dysfunction of the parasympathetic nerves on the basis of the results of pharmacological examinations,

\footnotetext{
* Correspondence to: Okamoto, Y., Department of Veterinary

Medicine, Faculty of Agriculture, Tottori University, 4-101

Koyama-minami, Tottori 680-8553, Japan.

e-mail: yokamoto@muses.tottori-u.ac.jp
}

and we report herein the clinical features, diagnosis, and treatment.

The first case, a 5-year-old intact female Miniature Dachshund, was referred with photophobia to the Tottori University Veterinary Teaching Hospital, Japan. The results of a physical examination were unremarkable, except for photophobia. An ocular examination revealed that the dog's left pupil was dilated and unresponsive to light (Fig. 1-A). The right pupil had a slightly smaller diameter than the left pupil. However, the direct and indirect responses to light were weak or negative bilaterally. The palpebral reflex was confirmed bilaterally. The results of Schirmer's tear tests were within the normal range at the first consultation [7]. The results of other ocular examinations, including fundoscopy and measurement of intraocular pressure $(17 \mathrm{~mm}$ Hg OD, 17 mm Hg OS), were also unremarkable, and all ocular structures were normal. Computed tomography of the dog's head did not reveal any abnormalities that might explain the clinical features.

The second case was a 7-year-old intact male Shih Tzu was referred with mydriasis and photophobia. No abnormalities were detected on general physical examination. An ocular examination revealed that both pupils were dilated and unresponsive to light. We confirmed normal palpebral reflex bilaterally. The results of Schirmer's tear test and the dog's intraocular pressure (15 mm Hg OD, $20 \mathrm{~mm} \mathrm{Hg}$ OS) were within the normal range at the first consultation [6].

In both cases, there were no clinical symptoms, history, or minimum database that made us suspect an important differential diagnosis (e.g., diabetes, hyperthyroidism, myasthenia gravis, or tumor). The owners did not know when the abnormal mydriasis and photophobia had first occurred. We observed that these dogs had no problems with their vision or behavior. We also confirmed the menace and daz- 

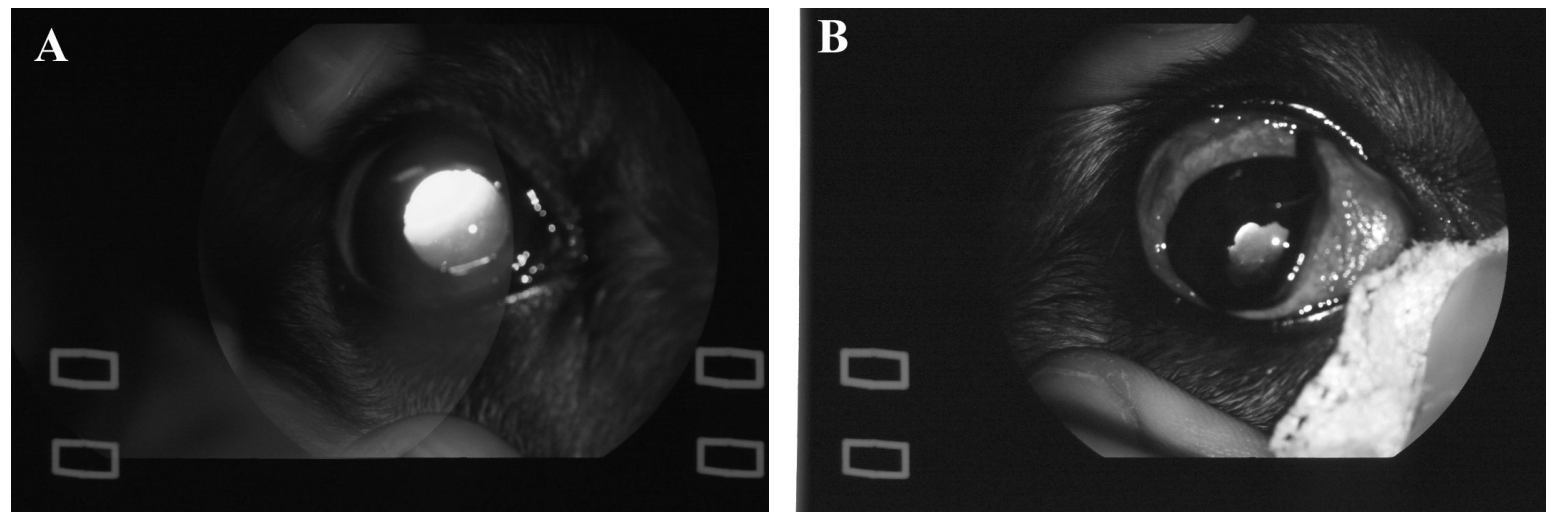

Fig. 1. Mydriasis and miosis in the first case. The pupil was dilated and unresponsive to light (A). After administration of $0.1 \%$ pilocarpine, the diameter of the pupil became smaller (B).

zle reflexes in both cases. The cotton ball test also indicated no abnormalities. Thus, we did not perform an electroretinogram examination.

According to previous reports, we performed pharmacological examination using $0.1 \%$ pilocarpine (Sanpilo $2 \%$, Santen Pharmaceutical Co., Ltd, Osaka, Japan; diluted to $0.1 \%$ with eyewash) with a single drop applied to each eye $[2,5,9]$. After application, we monitored the eye's response every $5 \mathrm{~min}$ for $60 \mathrm{~min}$. Pupil size was measured directly with a ruler. This test is used to diagnose parasympathetic denervation of the affected pupillary sphincter muscle.

In the first case, $0.1 \%$ pilocarpine administration resulted in miosis of the right pupil for $35 \mathrm{~min}$ and the left pupil for 55 min (Fig. 1-B). Additionally, blepharospasm and prolapse of the nictitating membranes were observed bilaterally within 10 min after administration of the solution. The $2 \%$ pilocarpine solution also resulted in miosis in the first case. After administration of $2 \%$ pilocarpine, the pupillary margin seemed to be irregular (Fig. 1-B). This suggested that there was not only pharmacological dysfunction but also another abnormality. We could not find the reason for the irregular pupil, such as iritis. In the second case, there was no response to $0.1 \%$ pilocarpine in either pupil for $2 \mathrm{hr}$ after administration. However, $2 \%$ pilocarpine induced obvious miosis bilaterally. This indicated a topical parasympathetic lesion but did not suggest neuroanatomic localization [4]. On the basis of the miosis induced by pilocarpine, we diagnosed both dogs with a pupillary abnormality caused by parasympathetic dysfunction but not primary iris lesions.

After diagnosis, the dogs were treated with prednisolone (1-2 mg/kg, PO, SID) for 1 week to rule out inflammatory and autoimmune disorders. However, these treatments did not improve the clinical features. At the owner's request, we performed no further examination and treatment in the first case. The second case has been followed-up for over 240 days without clinical changes.

Abnormalities of the pupillary sphincter or pupillary dilator or dysfunction of the sympathetic or parasympathetic nerves innervating these muscles are the primary mecha- nism of pupillary abnormality. This mechanism is common to dysautonomia because the cases of canine dysautonomia also cause mydriasis and prevent miosis after administration of $0.05 \%$ or $0.1 \%$ pilocarpine [6]. Dogs affected with dysautonomia have been reported to exhibit various clinical signs, including vomiting, diarrhea, depression, inappetence, reduced or absent anal tone, dysuria, and mydriasis. However, in both our cases, there were no such clinical signs, except for mydriasis. Therefore, we ruled out dysautonomia. In addition, a lack of deep tendon reflexes is not consistent with the condition described in Adie's syndrome in humans.

In both cases, miosis was induced by $2 \%$ pilocarpine used as a muscarinic receptor agonist. This result confirmed a topical neurologic lesion but did not allow for neuroanatomic localization to the central, preganglionic, or postganglionic regions [4]. Primary pupillary lesions were also ruled out. Additionally, in the first case, $0.1 \%$ diluted pilocarpine induced miosis in both pupils. Such a low concentration of pilocarpine does not induce miosis in normal pupils [4]. This result indicates that denervation had rendered the parasympathetic nerves supersensitive to the muscarinic receptor agonist. Therefore, abnormal mydriasis in the first case was thought to have been induced by denervation of the postganglionic parasympathetic nerves innervating the pupillary sphincter [1].

The parasympathetic preganglionic neurons of cranial nerve III are located in the rostral extension of the EdingerWestphal nucleus, ventral tegmental area, and anteromedial nucleus [4]. These preganglionic fibers branch off the central nervous system and synapse in the ciliary ganglion with postganglionic neurons that innervate the pupillary sphincter. In addition, the cholinergic parasympathetic postganglionic neuron, a short ciliary nerve, releases acetylcholine, which binds to muscarinic receptors at the neuroeffector junction. In the first case, the connection between the short ciliary nerve and pupillary sphincter corresponded to the neuroeffector junction. Thus, the result of pharmacological testing using $0.1 \%$ pilocarpine suggested dysfunction of the 
ciliary ganglion, short ciliary nerve, or neuroeffector junction between the short ciliary nerve and pupillary sphincter. In some other cases of total oculomotor nerve palsy, mydriasis has been observed because of such anatomic specificity. We could not find ptosis and internal or external ophthalmoplegia caused by a dysfunctional oculomotor nerve in either of our cases. Thus, we ruled out oculomotor nerve palsy, and concluded that the cause of this mydriasis was dysfunction of the parasympathetic nerves on the basis of the clinical signs and results of pharmacological testing. However, we could not determine the neuroanatomic localization of the lesion in the second case. The result of pharmacological test using $2 \%$ pilocarpine ruled out the possibility of a primary pupillary lesion. To obtain further diagnostic information, magnetic resonance imaging of the dog's head would have been required.

In humans, immune-mediated autonomic neuropathy, systemic autoimmune disorders, infection, hereditary neuropathic disorders, neurodegenerative disorders, nutritional deficiency, toxic agents, and diabetes have been reported to cause tonic pupils [8]. Intracranial meningioma has been reported to cause tonic pupils in 2 dogs $[5,9]$. However, our cases had no other clinical features except for mydriasis, and they had no history of disease. Thus, we could not determine the cause of parasympathetic nerve dysfunction in these dogs. However, it was unlikely that inflammatory, immune- or autoimmune-mediated mechanisms were involved in either case, because we did not obtain any improvement after oral administration of prednisolone. We observed bilateral blepharospasm and prolapse of the nictitating membranes in the first case after administration of pilocarpine. These reactions are consistent with a topical irritation caused by the low $\mathrm{pH}$ of pilocarpine but not a pharmacologic effect.

Most cases of primary abnormal pupil function do not cause clinical problems. Dilated pupils as described here can result in photophobia. Abnormalities of pupil function may be a sign of ophthalmic disease. It is important to perform a thorough ocular examination when presented with abnormal papillary responses. In some cases, abnormal mydriasis or miosis may be a sign of a systemic problem. Veterinarians should perform thorough general clinical and ophthalmic examinations and endeavor to make a correct diagnosis by taking these abnormalities into account.

The authors would like to thank Professor Dr. Dennis E. Brooks, College of Veterinary Medicine, University of Florida, for his valuable suggestions and encouragement.

\section{REFERENCES}

1. Collins, B.K. and O'Brien, D. 1990. Autonomic dysfunction of the eye. Semin. Vet. Med. Surg. 5: 24-36.

2. Gerding, P.A., Brightman, A.H. and Brogdon, J.D. 1986. Pupillotonia in a dog. J. Am. Vet. Med. Assoc. 189: 1477.

3. Kern, T.J., Aromando, M.C. and Erb, H.N. 1989. Horner's syndrome in dogs and cats: 100 cases (1975-1985). J. Am. Vet. Med. Assoc. 195: 369-373.

4. Klauss, G. and Constantinescu, G.M. 2004. Nonhypotensive autonomic agents in veterinary ophthalmology. Vet. Clin. North Am. Small Anim. Pract. 34: 777-800.

5. Larocca, R.D. 2000. Unilateral external and internal ophthalmoplegia caused by intracranial meningioma in a dog. Vet. Ophthalmol. 3: 3-9.

6. Niessen, S.J., Eastwood, J., Smyth, J.B. and Cherubini, G.B. 2007. Five cases of canine dysautonomia in England (2004 to 2006). J. Small Anim. Pract. 48: 346-352.

7. Strubbe, D.T. and Gelatt, K.N. 1999. Ophthalmic examination and diagnostic procedures. pp. 456-457. In: Veterinary Ophthalmology, 3rd ed. (Gelatt, K.N. ed.), Lippincott Williams \& Wilkins, Baltimore.

8. Toth, C. and Fletcher, W.A. 2005. Autonomic disorders and the eye. J. Neuro-Ophthalmol. 25: 1-4.

9. Webb, A.A., Cullen, C.L., Rose, P., Eisenbart, D., Gabor, L. and Martinson, S. 2005. Intracranial meningioma causing internal ophthalmoparesis in a dog. Vet. Ophthalmol. 8: 421425. 